CRS) and aspirin (4h ASP) induced ulceration. The ulcer index and \% protection was calculated. The gastric juice collected from $4 \mathrm{~h}$ PL rats was studied for the effects on gastric volume, $\mathrm{pH}$, free acid, total acid-pepsin output, DNA gastric juice cell shedding and mucosal cell proliferation. Biochemical estimation such as histamine content, mucus content and $\mathrm{H}^{+} \mathrm{K}^{+}$ATPase inhibitory activity was also evaluated. The effect of APF on stomach histopathology was also studied. Statistical analysis was done by one way ANOVA followed by Dunnett's post hoc test for multiple comparisons against control group. The difference was considered to be significant when $P<0.05$. Oral treatment of APF (200 and $400 \mathrm{mg} / \mathrm{kg}$, p. o.) for 7 days showed significant protection against acute gastric ulcer induced by $4 \mathrm{~h} P \mathrm{PL}$ and $95 \% \mathrm{EtOH}$, but was found to have no effect on CRS and ASP-induced gastric ulceration. The gastric studies showed that treatment with APF signi cantly increased the gastric juice $\mathrm{pH}$, decrease the gastric volume and total acid-pepsin output and significantly reduced the gastric juice cell shedding. A significant decrease in histamine content and a reduction in free phosphate ions responsible for $\mathrm{H}^{+} \mathrm{K}^{+}$ATPase inhibitory activity were also observed. In conclusion, the results of the present study indicate that the gastroprotective activity of APF contributes mainly to the peripheral effects on the gastric secretion and the probable mechanism underlying behind such activity might be both due to the possible inhibition on the histaminic $\left(\mathrm{H}_{2}\right)$ receptor and $\mathrm{H}^{+} \mathrm{K}^{+}$ATPase pump.

\title{
INVESTIGATION OF THREE TRITERPENE ACIDS AGAINST CANCER CELL PROLIFERATION ACTIVITY
}

\section{○ Khoo Gaik Ming, Clausen Morten Rahr, Larsen Erik}

Department of Food Science, Aarhus University, Kirstinebjergvej 10, DK-5792 Aarslev, Denmark

The object of this study was to investigate the inhibitory effect of betulinic acid, oleanolic acid and ursolic acid on human colon cancer cell proliferation activity. Triterpene acids are members of the terpene family and are widely distributed in food plants. A rosehip fraction containing a mixture of betulinic acid, oleanolic acid and ursolic acid displayed inhibitory effect against the release of pro-inflammatory cytokine interleukin- 6 with the combination of oleanolic acid/betulinic acid and ursolic acid/betulinic acid displaying significant synergistic inhibitory effects (1). Our results showed that these three compounds also displayed good inhibitory effect against cancer cell proliferation activity at the concentration of $10 \mu \mathrm{g} / \mathrm{ml}$. both betulinic acid and ursolic acid displayed strong inhibition against HT-29 (88\% and $79 \%$, respectively) and SW-480 (76\% and $82 \%$, respectively), but a moderate to weak inhibition against Caco-2 (19\% and $22 \%$, respectively). Oleanolic acid displayed only moderate inhibition against Caco-2 (49\%), very weak inhibition against HT-29 (7\%) and no inhibition against SW-480. No synergistic effects were observed between combinations of the triterpene acids. We only found an additive effect in the combination of oleanolic acid/ursolic acid and only against proliferation of HT-29. Antagonistic effects between the pure compounds were observed when betulinic acid was one of the components in the combination. Although betulinic acid and ursolic acid showed good inhibitory effects against all three cancer cell lines, their combination displayed antagonistic effects and showed poor inhibition against cancer cell proliferations. However, these triterpenic acids and their combination effects should be further investigated in different in vitro or in vivo experiment to confirm their bioactivities and mechanism.

Reference: (1) Saaby L., Moesby L., Hansen E.W., Christensen S.B. (2011). Isolation of immunomodulatory triterpene acids from a standardized rose hip powder (Rosa canina L.). Phytotherapy Research, 25, 195-201.

\section{DECURSIN AND RELATED COMPOUNDS INHIBIT VASCULAR ENDOTHELIAL MIGRATION BY SPHINGOSINE KINASE INHIBITION}

\section{○ Lee Yong-Moon, Shin Kyong-Oh, Seo Cho-Hee, Kim Tack-Joong}

${ }^{1}$ College of Pharmacy, Chungbuk National University, Cheongju, South Korea

${ }^{2}$ Division of Biological Science and Technology, Yonsei University, Wonju, South Korea

The pyranocoumarin compound decursin isolated from the herb, Angelica gigas, are known to possess potent anti-inflammatory activities. However, little is known about their anti-angiogenic activity. Here, we investigated the anti-angiogenic effects of decursin by the changes of sphingolipid metabolites 